\title{
Accompagner et apprendre par les pairs : une expérience de rédaction de $\mathrm{CV}$
}

\author{
VALÉRIE BILLAUDEAU \\ ESO-Angers UMR CNRS 6590 Espaces et Sociétés, Université d'Angers \\ valerie.billaudeau@uni-angers.fr \\ NATHALIE DEBSKI \\ Université d'Angers \\ nathalie.debski@univ-angers.fr
}

\section{Résumé}

Cette communication propose une démarche originale d'apprentissage par les pairs dans une relation interdisciplinaire. Cette démarche a porté sur la réalisation de $\mathrm{CV}$ et a été conduite au cours de quatre années à l'Université d'Angers entre des étudiants ingénieurs de l'ISTIA et les étudiants en alternance de Master 2 Ressources Humaines et Organisations Innovantes (RHOI). Fondant notre démarche sur les travaux d'accompagnement et d'apprentissage par les pairs, nous avons fait l'hypothèse que cette activité co-élaborée, où chacun joue un rôle actif, mobiliserait un intérêt commun pour les deux parties prenantes et favoriserait un meilleur résultat que l'unique séance de cours de l'enseignant. À partir des retours d'expérience d'environ 400 étudiants ingénieurs et 60 étudiants de master 2 en RHOI, plusieurs effets positifs ont été constatés : le travail entre pairs a facilité les échanges entre les étudiants malgré leur emploi du temps peu compatible et chargé (les masters 2 étant en alternance). Il a permis aux pairs de s'approprier plus facilement le contenu avec un apport considérable des deux côtés. Par ailleurs, cette expérience a développé une co-responsabilisation des étudiants du fait des enjeux professionnels perçus par chacun influençant leur implication. Enfin la qualité de l'apprentissage est validée par les professionnels qui ont remarqué l'évolution des CV depuis la mise en place de cette organisation.

\footnotetext{
Abstract:

This study was undertaken during 4 years at the University of Angers based on an innovative multi-disciplinary teaching approach. The idea was to get engineering students at ISTIA
} 
(Angers University) and masters students currently in apprenticeship (masters in Human Resources) help one another write up their CV. Our method is to let peers work and learn together based on the assumption that cooperation would bring about a more active student participation and that shared interest would work better than a teacher-delivered course. The feedback of 400 student engineers and 60 masters student was positive. Working together has eased exchanges between students despite their busy agendas, especially for those in apprenticeship. Students really grasped the matter with input from both sides. This experiment has developed a feeling of shared responsibility regarding what is at stake for them professionally and each student understood essential to reach the goal. It has been successfully recorded by professionals who noticed the improvement in CVs.

\section{Mots-clés}

accompagnement par les pairs, apprentissage, curriculum, interdisciplinarité, transformation

\section{Key words}

peers work, learn together, curriculum, interdisciplinarity, transformation

\section{Introduction}

Rédiger un curriculum vitae est une étape essentielle dans la recherche professionnelle des étudiants qui se préparent au marché de l'emploi. Plusieurs difficultés se présentent à eux pour préparer ce document : la valorisation de leurs expériences qui ne leur semblent pas significatives, la nécessité de préciser un projet professionnel et la complexité de créer un CV répondant aux codes attendus tout en le personnalisant et le différenciant. Pour ceux qui accompagnent cette démarche, les difficultés ne sont pas moindres : il faut faire connaissance avec son interlocuteur, sa personnalité, ses expériences, saisir ce qui le caractérise et ce vers quoi il veut aller, le guider sans imposer son point de vue, découvrir des professions, des secteurs d'activités, se mettre à la place des recruteurs, rester dans l'actualité des besoins. Pour ce faire, de nombreux sites internet donnent des conseils, des vidéos sur YouTube abordent la question en images et en son et des plateformes mettent à disposition des modèles (moncvparfait.com, doyoubuzz.com, primocv.com, etc.). Ce foisonnement d'informations constitue une aide mais ne rassure pas pour autant les futurs professionnels. 
Au sein de l'ISTIA-école d'ingénieurs de l'Université d'Angers, une centaine d'étudiants de seconde année (EI4) bénéficient d'un module de formation pour l'entraînement à l'entretien de recrutement ; ils doivent rédiger leur $\mathrm{CV}$ et une lettre de motivation adaptée à une offre de stage en vue de se préparer au futur échange avec des recruteurs lors de la journée annuelle des métiers. Après dix années d'encadrement du module à la rédaction des $\mathrm{CV}$, le constat de l'enseignant référent est une récurrence des mêmes défauts et l'insatisfaction d'un suivi peu individualisé du fait du très grand nombre de $\mathrm{CV}$ à traiter. Un accompagnement par les pairs a alors été proposé.

Dans le cadre de cette communication, nous concentrerons notre attention sur la création du CV qui a fait l'objet d'une démarche originale d'apprentissage par les pairs dans une relation interdisciplinaire. L'expérience menée à l'Université d'Angers a mis en relation, durant quatre années (2013-2016), les étudiants ingénieurs de l'ISTIA et les étudiants en alternance de master 2 Ressources Humaines et Organisations Innovantes (RHOI). Chaque année, 20 étudiants se spécialisant en Management des Ressources Humaines ont accompagné chacun 5 à 6 futurs ingénieurs dans la création et/ou l'amélioration de leur $\mathrm{CV}$, par des conseils de structuration, d'organisation et de valorisation de leurs compétences de pairs à pairs, tout en ayant une pratique réflexive sur ce qui a fait sens dans la relation et pour le pair accompagnateur.

Cette démarche d'apprentissage avec deux formations d'une même université pose la question de l'altérité : Quels types de relations se nouent entre eux ? Qu'est-ce que chacun en retire ? En définitive, est-ce qu'un transfert entre étudiants peut être fructueux dans une perspective d'insertion professionnelle?

Nous avons fait l'hypothèse que les étudiants du Master 2 RHOI pouvaient apporter leurs compétences aux étudiants ingénieurs de l'ISTIA tout en développant une autonomie et une posture professionnelle, et ce pour deux raisons. D'une part, parce qu'ils sont tous en alternance dans les services de ressources humaines d'entreprises ${ }^{1}$, et sont donc confrontés au recrutement et à la sélection de CV. D'autre part, parce qu'ils ont été eux-mêmes en situation de réalisation de leur propre $\mathrm{CV}$ pour obtenir un contrat de professionnalisation nécessaire à leur formation.

\footnotetext{
1 Edf, La Poste, Eram, Chu d'Angers, Expectra, Seb Moulinex, Terrena, Scania, La Boucherie, Orange, Sncf, MSA, Elivia, Pasquier, Strego, KPMG, GEVES, Action contre la faim, etc.
} 
Après une revue de la littérature sur l'accompagnement par les pairs, nous présenterons la méthodologie mise en place ainsi que les résultats obtenus quant au degré d'implication des étudiants et leur niveau de satisfaction en étant devenus parties prenantes de leur formation. La discussion et les perspectives permettront de saisir les freins et les limites de cette expérience et les possibilités qu'elle a offertes au niveau pédagogique sur la question du recrutement.

\section{Accompagner et apprendre par les pairs}

\subsection{Un cheminement conjoint...}

La revue de la littérature sur l'accompagnement par les pairs nous montre que ce concept est à la fois protéiforme et polysémique. Pour Maela Paul (2004, 2007), l'accompagnement peut être réalisé sous forme de conseil, de coaching, de parrainage, de mentorat, de tutorat, ou encore de compagnonnage individuel ou collectif ${ }^{2}$. Il implique une posture relationnelle entre deux ou plusieurs individus et prend la forme d'un cheminement conjoint dans lequel l'incertitude a sa place. Il débouche alors sur une co-construction. Mais l'accompagnement a également une fonction support qui permet une mobilisation de ressources entre ces individus dans un contexte d'accès et de développement du savoir. Pour l'auteure, il faut donc se mettre à deux pour accompagner afin de porter un autre regard, faire un pas de côté et engager une démarche réflexive, une méta-réflexion._Mais revenons d'abord sur ce qu'est un pair, cet « autre » qui n'est pas moi mais qui me ressemble.

\subsection{De pair à pairs}

Est un pair « la personne de même situation sociale, de même titre, de même fonction qu'une autre personne » et « celle qui occupe le même rang, qui est égale en dignité, en situation sociale» ${ }^{3}$, c'est un égal. Ce dernier peut appartenir à la même année d'étude, à la même formation mais à des âges et expériences différents, ou encore à des formations différentes mais

\footnotetext{
2 Nous ne reviendrons pas ici sur la définition de chacun des termes et renvoyons le lecteur aux travaux de Maela Paul (2004, 2007). Sur le compagnonnage, le lecteur pourra suivre les travaux d'Annabelle Hulin (2010).

3 Définition du Centre National de Ressources Textuelles et Lexicales www.cnrtl.fr et du Larouse www.larousse.fr/dictionnaires/francais/pairs
} 
au statut identique dans la même université. Enfin, il peut appartenir à des formations différentes au statut identique dans des universités différentes.

De nombreux travaux montrent que si l'accompagnement par les pairs est une alternative intéressante au cours magistral, le bénéfice en termes d'apprentissage n'est pas automatique. Une interdépendance sociale positive est nécessaire ; c'est-à-dire une situation dans laquelle les individus partagent un but commun, et le résultat de chacun est affecté par les actions des autres (Deutsch, 1949a, 1949b, 2006 ; Johnson et Johnson, 1989, 2003). De même, une coconstruction des connaissances est plutôt favorable à l'accompagnement. Ainsi, lorsqu'un étudiant essaie de faire comprendre à un autre étudiant, il offre la possibilité à ce dernier de poser des questions et de donner son avis. Ces interactions augmenteraient le traitement profond des informations pour l'ensemble des participants (O'Donnell et King, 1999). De ces différentes interactions naîtraient un apprentissage par les pairs.

\subsection{De la transmission à la transformation}

Au-delà d'une simple transmission s'opère ainsi une forme de déplacement et de transformation plus complexe chez chaque partie-prenante. Les travaux de Jeanne Thébault (2013) montrent notamment que « la transmission est souvent considérée comme un phénomène allant de soi » par simple côtoiement, reposant avant tout sur les caractéristiques individuelles des protagonistes ; l'un, plus ancien dans l'organisation ou l'entreprise et plutôt âgé, pouvant « transférer ses savoirs » vers un nouveau, généralement plus jeune.

Or, la transmission relève d'un processus qui se construit au fil du temps. Pour Thébault et al. (2014), elle est élaborée par les individus et doit s’intégrer à l'activité de production et est alors circonstancielle et opportuniste. Elle est également une activité co-élaborée, où chacun joue un rôle actif. Celui du pair-accompagnateur est d'aider, d'accompagner le nouveau ou novice pour des tâches qu'il n'est pas en mesure de réaliser seul. Toutefois, le pair-accompagné contribue aussi à orienter les interventions de l'accompagnateur en donnant des informations sur ses propres connaissances et en posant des questions. 


\subsection{Apprentissage et autonomisation}

Cette transformation par l'accompagnement par les pairs débouche ainsi sur le concept d'apprentissage par les pairs qui, selon Mazur (1997), est un style d'apprentissage interactif dans lequel les apprenants sont très actifs dans le processus d'apprentissage. Il repose sur un esprit de partage et de collaboration pour résoudre une situation-problème précise, les apprenants renforçant alors leurs apprentissages et contribuant par ailleurs à la construction de ceux de leurs pairs.

Cette idée de partage se rapproche de la théorie du don conceptualisée par Marcel Mauss (1968) selon laquelle le don s'opère dans un échange en principe illimité don/contre-don/contre-contredon/etc., qui s'effectue à partir d'une triple obligation, celle de donner, de recevoir et de rendre. Ce type d'échange produit alors beaucoup plus de liens sociaux que l'échange contractualisé. Les travaux de Fustier (2012) vont dans ce sens et dans le cas de l'accompagnement par les pairs, les parties échangent des savoirs en construction ; don d'une connaissance-référence, contre-don de connaissance à intégrer, contre-contre don de transformer la connaissance initiale en compétence de type savoir-faire.

L'apprenant est désormais un partenaire actif du dispositif, non un simple consommateur. Le lien social a créé de la valeur pour les deux parties prenantes et a transformé leurs propres connaissances sur le problème à résoudre.

Cet apport conceptuel nous a alors conduites au travers de l'expérimentation suivante à mettre en lien l'accompagnement par les pairs et la transformation des connaissances de nos étudiants dans la construction de leur CV.

\section{Expérimentation et méthodologie}

Depuis quatre ans, nous avons mis en place un apprentissage entre pairs pendant un mois et demi en donnant aux étudiants un cadre tout en leur laissant de l'initiative et de l'autonomie.

\subsection{De l'expérimentation...}

L'apprentissage par les pairs nécessite un travail préparatoire important pour l'enseignant mais aussi pour les parties prenantes. 


\subsubsection{Préparer les apprentissages auprès des étudiants ingénieurs}

Les étudiants de l'ISTIA bénéficient d'une dizaine d'heures pour s'entraîner à la simulation d'entretien de recrutement qui se fait en janvier. Il leur faut préparer un $\mathrm{CV}$, choisir une offre de stage et écrire une lettre de motivation adaptée. Une seule séance de travaux dirigés (TD) est consacrée au CV puisqu'il ne constitue qu'une étape pour atteindre l'objectif de la simulation d'entretien de recrutement. En 1 heure 20 (durée du TD), trois aspects sont abordés : nous rappelons les fondamentaux du $\mathrm{CV}$ et échangeons les pour et les contre de certains choix (photo ou pas photo, par exemple). Nous utilisons la plateforme Moodle pour un temps où les étudiants vont lire la synthèse préparée par l'enseignant et prendre connaissance des pièges relevés dans les $\mathrm{CV}$ à partir des travaux des années passées. Enfin, les étudiants reprennent leurs CV (préparés pour la séance) en binôme afin de détecter si des erreurs « classiques » ne parsèment pas leurs documents. Ils ont enfin pour mission de rectifier leur $\mathrm{CV}$, réfléchir à la façon dont ils vont se présenter pour le prochain rendez-vous qui consiste à rencontrer leur futur pair accompagnateur RH : les étudiants du master 2 RHOI.

\subsubsection{Donner le « juste ce qu'il faut » aux étudiants pairs-accompagnateurs}

Les étudiants du master 2 RHOI bénéficient d'un temps de présentation de leur mission avec l'enseignant des étudiants de l'ISTIA et leur responsable pédagogique. À cette occasion, les objectifs du travail leurs sont présentés. Ils s'articulent en deux grands points : 1- les étudiants RHOI ont le rôle de pair-accompagnateur, c'est-à-dire qu'ils ont à organiser leur accompagnement en fonction de leur pair-accompagné et à apporter une valeur ajoutée pour chaque étudiant afin d'arriver à un résultat qui satisfassent les deux parties prenantes ; 2- les étudiants ingénieurs sont les « commanditaires » et peuvent être plus ou moins motivés par leur accompagnement : les étudiants RHOI ont à relever le défi de montrer l'importance de leur présence et de leurs compétences.

Cette rencontre permet de répondre aux questions et de présenter les spécialités de l'école d'ingénieurs de l'ISTIA qui coïncident avec les débouchés professionnels des étudiants. En général, bien que l'ISTIA fasse partie de l'Université d'Angers, les Masters 2 RHOI ne connaissent pas ces formations et encore moins les débouchés possibles. Ainsi, ils découvrent que l'ISTIA forment aux systèmes automatisés et en génie informatique, à la qualité, l'innovation et la fiabilité, aux bâtiments et à la sécurité (et bientôt au génie biologique et santé). 
Ils poursuivent cette introduction par des recherches personnelles afin de réunir les éléments qui leurs sont utiles pour le temps de face à face avec les étudiants ingénieurs.

\subsubsection{Lancement vers l'autonomie}

Les étudiants étant préparés de part et d'autre, une séance commune est organisée afin qu'ils fassent connaissance et mettent en place leurs modalités de fonctionnement. En plénière, les enseignants rappellent les objectifs avant que les pairs-accompagnateurs se présentent et partent dans une salle avec les étudiants qu'ils vont suivre. Certains pairs-accompagnateurs ont prévu une présentation sur les $\mathrm{CV}$, d'autres commencent par un tour de table et regardent directement les CV des pairs-accompagnés de l'ISTIA, d'autres encore ont organisé un ordre de passage individuel pour chaque pair-accompagné : en bref, les pairs-accompagnateurs spécialistes en ressources humaines mettent en place leur dispositif d'accompagnement à partir de la stratégie qu'ils ont élaborée en amont. De leur côté, les étudiants ingénieurs sont piqués par la curiosité et très intéressés pour échanger entre eux et comparer la manière dont les étudiants du Master 2 RHOI ont procédé. À la sortie de ces rencontres, les échanges sont nombreux sur leurs impressions, les conseils donnés et les différences relevées.

\section{2. À l'évaluation}

L'enseignant de l'ISTIA n'intervient pas dans l'accompagnement du CV durant toute la durée (de fin septembre à novembre) du travail mené entre les étudiants du Master 2 RHOI et les étudiants ingénieurs. Il rencontre cependant les étudiants de l'ISTIA chaque semaine lors des TD et rappelle les délais, notamment celui de la future journée des métiers (qui a lieu en novembre), pour laquelle il est nécessaire d'avoir un CV finalisé. Plusieurs évaluations sont organisées. Du côté des étudiants ingénieurs de l'ISTIA, le CV doit être prêt pour les délais impartis et fait partie de la note attribuée lors de la simulation à l'entretien de recrutement en janvier. Ils ont également un questionnaire de « satisfaction » à remplir sur l'accompagnement dont ils ont bénéficié qui permet aussi d'identifier leurs apprentissages. Les masters 2 RHOI, quant à eux, sont évalués sur le bilan du travail réalisé avec les étudiants accompagnés ainsi que sur leur réflexivité de leur accompagnement. 


\section{Méthodologie}

Pour vérifier l'efficacité de cette expérimentation, nous avons mobilisé les résultats de nos deux collectes de données. Le questionnaire de satisfaction des étudiants de seconde année d'école d'ingénieur (EI4) a été rempli par chaque étudiant ingénieur lors de la dernière séance de préparation à l'entretien de recrutement. Ainsi, chaque promotion de 100 étudiants a renseigné quinze questions à choix multiples concernant les apports transmis par leur pair. En parallèle et à l'issue de leur accompagnement, les Master 2 RHIO ont réalisé un bilan individuel de cette expérience à partir de six questions ouvertes desquelles nous tirons les verbatim : (Quels ont été les apports de cette expérience ? Quelles ont été les difficultés rencontrées ? Quels ont été les éléments de satisfaction et d'insatisfaction? Qu'est-ce que vous referiez de la même façon ou différemment? Quelles sont vos suggestions pour un travail plus satisfaisant? Seriez-vous prêt à réitérer ce coaching (oui pourquoi, non pourquoi ?)). Les résultats présentés ci-après proviennent donc de résultats quantitatifs collectés auprès d'environ 400 étudiants ingénieurs et qualitatifs auprès de 60 étudiants de Master 2.

\section{Résultats}

Après une première année d'expérimentation encourageante au regard des résultats, nous avons poursuivi ce travail entre pairs entre 2013 et 2016. Voyons les résultats de cette expérimentation.

\subsection{Satisfaction des étudiants ingénieurs}

La majorité des étudiants ingénieurs est très satisfaite (plus de $80 \%$ ), dont $33 \%$ considèrent les conseils pertinents et $16 \%$ en phase avec leurs attentes. Ils sont par ailleurs convaincus d'avoir amélioré leur CV grâce aux apprentissages transmis par leurs pairs. Leurs acquis peuvent être classés en deux grandes catégories : 1. la structure du CV avec une attention particulière au degré de précision, de concision et de vocabulaire ; 2. la mise en valeur du projet professionnel à travers les expériences et les compétences. Ainsi, les verbatim témoignent d'apprentissages simples et déjà connus sur les sites internet : «mettre une photo avec un grand sourire », «séparer l'expérience professionnelle et l'emploi saisonnier », «structurer mon 
$\mathrm{CV} »$, utiliser un vocabulaire adéquat », «être pointilleux », etc. Ces évidences et conseils présentés partout (ouvrages, revues, internet, etc.), ainsi que par leur enseignant, ne garantissent pas le résultat. Alors que le système d'aller-retour entre les étudiants ingénieurs et ceux de master RHOI permet de personnaliser les remarques pour chaque étudiant ingénieur et de revenir sur ces présupposés. De plus, cette pédagogie entre pairs offre surtout l'intérêt d'approfondir les points délicats que sont la mise en valeur des expériences et des compétences. Les étudiants ingénieurs le perçoivent particulièrement et reconnaissent que les échanges avec les étudiants du Master 2 RHOI ont permis de « mieux arriver à se vendre », « organiser plus efficacement mon $\mathrm{CV}$ avec les détails sur les expériences », « accorder une place importante à l'accroche ».

Une relation de confiance s'instaure entre les étudiants bien qu'une année seulement les sépare dans leur formation. Ceux qui ont acquis tout le crédit aux yeux des étudiants ingénieurs, sont sollicités pour aller plus loin que le contrat de départ initialement prévu. En effet, $15 \%$ des étudiants ingénieurs de l'ISTIA demandent des avis sur leur lettre de motivation ou l'entretien de recrutement. Au final, les étudiants du Master 2 RHOI génèrent des impressions très positives : «c'était très bien comme initiative !», « Recommencer l'an prochain ! », « Pour ma part, j'ai gardé mon coach en contact Skype. », « Il m'a dit que si j'avais besoin, je pouvais le recontacter », «Ma coach a été parfaite, d'ailleurs nous restons toujours en contact», «Mon coach était une personne très dynamique et réactive, très pointilleuse que je remercie ». $80 \%$ des étudiants ingénieurs ont pensé à remercier leur coach par écrit...

Néanmoins, $8 \%$ des étudiants ingénieurs font un retour négatif sur leurs échanges avec les étudiants du Master 2 RHOI car ils estiment ne pas avoir appris plus de choses que ce qu'ils ne savaient déjà, ces derniers provenant d'un DUT où la question du CV est déjà abordée.

\subsection{Bilan des étudiants du Master 2 RHOI}

Les étudiants du Master 2 RHOI sont particulièrement satisfaits de leur travail lorsqu'ils ont pu échanger plus de deux fois minimum avec l'étudiant ingénieur de l'ISTIA; ils peuvent ainsi voir l'évolution du CV. Toutefois, leur insatisfaction est forte quand ils ont pris le temps d'étudier scrupuleusement le CV et envoyé leurs recommandations sans voir le résultat produit. Cette frustration concerne environ $10 \%$ des étudiants ingénieurs de 1'ISTIA. 
Par ailleurs, les étudiants du Master 2 RHOI sont particulièrement satisfaits quand l'étudiant(e) est «agréable », « investi(e)», quand « les conseils sont pris en compte » et les étudiants ingénieurs « reconnaissants ». En général, tous les étudiants du Master 2 RHOI trouvent cette expérience «enrichissante », «bénéfique », « positive », tant d'un point de vue humain que professionnel ou tant pour eux que pour les étudiants de l'ISTIA. Le fait d'être autonome et responsable les fait d'abord douter de leurs capacités et ensuite gagner en confiance : «Ce projet m'a permis de gagner en légitimité puisque, malgré des doutes au départ, j'ai réussi à prodiguer des conseils RH personnalisés et pertinents à chaque étudiant. »

De plus, cette mission leur fait prendre du recul : «Je me suis rendu compte que certains conseils que je leur donnais étaient également valables pour mon propre CV », "Cela nous oblige donc à remettre en question nos pratiques, faire des recherches pour apporter des réponses aux points sur lesquels les avis divergent (exemple l'ordre et le contenu des rubriques...) et donc en apprendre davantage sur le sujet. » Cette expérience leur permet aussi d'acquérir des compétences en échangeant entre pairs dans la promotion : «Les étudiants m'ont posé des questions pertinentes notamment sur les points qui sont sujets à controverses au sein même de la « communauté » RH, tel que l'importance d'insérer ou non, une photo sur son CV. Cette base de réflexion a impulsé des débats ouverts et constructifs avec mes camarades de classe, qui m'ont aidé à me forger une opinion structurée sur les différents sujets abordés. »Cet accompagnement a été l'occasion de transmettre des connaissances « héritées d'une part de mon parcours académique, et d'autre part de mes expériences professionnelles, en particulier mon stage de $1^{\text {re }}$ année de master où je travaillais en qualité de recruteur » et « d'appliquer leurs acquis dans leur lieu de travail»; "j'ai également pu faire le lien avec les missions que j'effectue dans mon entreprise, pour tous mes recrutements j'ai pu mieux lire les nombreux CV qu'on reçoit et de faire le tri plus rapidement ». En définitive, et malgré quelques difficultés dont nous parlerons dans la discussion, les étudiants du Master 2 RHOI se sont sentis valorisés par cette démarche car ils ont eu le sentiment d'avoir « apporté de l'aide », d'avoir été « utiles » tout en apprenant eux-mêmes. 


\section{Discussion}

Avec cette pédagogie basée sur l'expérience et l'apprentissage par les pairs, nous avons tenté de mettre en relation deux types d'apprenants de formation et d'expériences différentes au sein d'une même université : les étudiants ingénieurs et les étudiants du Master 2 RHOI. Dans ce cadre, le rôle de l'enseignant ne s'est plus cantonné à transmettre la connaissance mais il devient celui de chef d'orchestre (Chevalier et Adjedj, 2014, p. 29). Cela rejoint les dispositifs de la classe inversée telle que le groupe PédagInnov l'a expérimenté en réfléchissant «à la façon dont on pourrait solliciter plus et mieux les savoirs préalables et l'énergie des apprenants » (Chevalier et Adjedj, 2014, p. 27). Ainsi, après avoir détecté les besoins et les compétences des parties prenantes, l'enseignant les a mises en relation avec un cahier des charges puis les a accompagnées dans l'autonomie avant de les évaluer.

À partir de cette expérience, nous avons constaté que le travail entre pairs a facilité les échanges, a permis aux pairs de s'approprier plus facilement le contenu, a développé une coresponsabilisation des étudiants, une forte implication des parties prenantes et enfin une qualité de l'apprentissage validée par les professionnels. Ces derniers, lors de la journée des métiers de 1'ISTIA-école d'ingénieurs de l'Université d'Angers de novembre et auxquels participent les étudiants d'EI4, ont en effet relevé la qualité des CV depuis que ce travail entre pairs est réalisé. Certains EI4 ont même accédé à des stages grâce aux conseils que leur avaient prodigué leurs pairs RHOI ; «Fort de mes observations du monde professionnel pour les entretiens RH, je leur ai fait part de mes analyses et méthodes afin qu'ils détiennent les clés pour réussir leur entretien. Enfin, je leur ai prodigué des conseils pour construire et maîtriser leur identité numérique, une activité (presque) incontournable à l'ère du numérique ».

Nous retrouvons ainsi cette interdépendance sociale positive nécessaire à l'apprentissage par les pairs de Deutsch $(1949,2006)$ et Johnson et Johnson $(1989,2003)$ ainsi que la coconstruction des connaissances de O’Donnell et King (1999). Ce travail entre pairs a permis une transmission de savoir professionnel en allant au-delà du contrat initial impulsé par l'enseignant et une transformation des apprentissages que nous retrouvons dans les travaux de Thébault (2013). Le témoignage précédant illustre l'initiative prise par de nombreux étudiants du Master 2 RHOI d'attirer l'attention des étudiants ingénieurs sur leur identité numérique ainsi 
que leur participation active à cet exercice. Par ailleurs, la place laissée à l'informel a agi comme facteur d'émulation pour les étudiants ingénieurs qui n'ont pas hésité à demander des conseils au-delà du CV et pour les étudiants du master 2 RHOI à offrir généreusement des opportunités : « J'ai proposé à ceux qui le souhaitaient de transmettre leur candidature pour un stage à la centrale (entreprise où l'étudiant en Master 2 RHOI réalise son alternance) ce qui les a enchantés. » Ces échanges de don, contre-don, contre-contre-don, sont allés dans les deux sens car les étudiants du Master 2 RHOI sont nombreux à souligner la découverte du monde de l'industrie à travers l'expérience des étudiants ingénieurs : «J'ai ainsi pu découvrir des personnes faisant partie d'un univers professionnel différent du mien avec une culture pédagogique différente. Cela a été bénéfique pour mon expérience au sein du groupe Seb Moulinex. En effet, nous avons beaucoup de personnes issues de formation ingénieur au sein du site et je suis amenée à rechercher régulièrement ce type de profil. J'ai ainsi pu en apprendre un peu plus sur les formations proposées dans cette filière et les compétences qui peuvent en découler... Cette découverte me servira certainement dans mon avenir professionnel dans les Ressources Humaines car j'ai la volonté de poursuivre plutôt dans le secteur industriel. » Au-delà de la découverte d'un univers, une éthique professionnelle émerge dans la pratique des étudiants du Master 2 RHOI: «J'avais l'appréhension que mes conseils, recommandations apparaissent en quelque sorte pour des ordres, chose que je ne voulais pas car leurs $\mathrm{CV}$ devaient rester très personnels et correspondre à la manière dont ils l'imaginaient... Il a été difficile de dissocier les remarques qui relevaient de mes goûts personnels de celles qui relevaient des codes à respecter. En effet, je me suis rendue compte que certaines fois je n'étais pas forcément d'accord sur les choix des couleurs, la présentation adoptée... Mais comme le CV retranscrit le portrait de chacun, le candidat doit donc le personnaliser selon ses goûts pour que celui-ci soit réellement son reflet. »

Cette attention à la singularité des étudiants accompagnés proche de l'altérité, l'application réalisée par les étudiants du Master 2 RHOI à sélectionner les mots les moins polysémiques et les plus encourageants lors de leur retour aux étudiants ingénieurs et le respect des choix des CV finaux témoignent d'un saut cognitif important durant cette démarche. Ils ont fait ce « pas de côté » et entamé une métacognition dans un cheminement conjoint (Paul, 2007). Ces étudiants ont non seulement investi leur rôle de pair-accompagnant mais ont également pris, en peu de temps, un recul proche de l'expérience professionnelle de plusieurs années et ont fait évoluer leurs propres compétences professionnelles (Wittorski, 1996) : «ce qui est aussi 
difficile dans le coaching CV, c'est le fait qu'il n'existe pas de CV idéal. Chaque recruteur a son CV idéal en tête, et cela dépend aussi du domaine dans lequel on candidate ».

\section{Perspectives}

Dans cette étude, nous avons perçu le lien entre accompagnement par les pairs et apprentissage par les pairs dans une situation d'asymétrie d'expériences et de statuts entre des étudiants ingénieurs et des étudiants alternants de Master 2 en situation professionnelle. Mais nous n'avons pas évalué la confiance accordée par les étudiants ingénieurs aux étudiants de Master en Sciences Humaines et Sociales sous forme de comparaison sociale des compétences qui, selon Bourgeois et Buchs (2011), pourraient parasiter l'apprentissage.

De même, le recours au numérique comme outil de travail n'a pas fait l'objet de notre attention alors qu'il a une place importante dans le processus : l'emploi du temps des parties prenantes étant trop contraignant, les échanges ont lieu principalement par mail. Une minorité d'étudiants utilise la visio-conférence pour discuter malgré leurs regrets de ne pouvoir se rencontrer et bénécifier/proposer un meilleur accompagnement. Ce comportement interroge l'utilisation spontanée des formes pédagogiques numériques de la générations $Z$ : l'accompagnement individualisé semble nécessiter, à leurs yeux, une présence physique.

\section{Références bibliographiques}

Bourgeois, E. et Buchs, C. (2011). Conflits sociocognitifs et apprentissage en formation. Dans P. Caspar et P. Carré (dir.), Traité des sciences et techniques de la formation (p. 291-308). Paris : Dunod.

Chevalier, L. et Adjedj, P.J. (2014). Une expérience de classe inversée à Paris-Est. Technologie, 194, 26-37. https://eduscol.education.fr/sti/sites/eduscol.education.fr.sti/files/ressources/techniques/7911/ 7911-194-p26.pdf

Deutsch, M. (1949a). An experimental study of the effects of cooperation and competition upon group processes. Human Relations, 2(2), 199-231.

Deutsch, M. (1949b). A theory of cooperation and competition. Human Relations, 2(2), 129151. 
Deutsch, M. (2006). Cooperation and competition. Dans M. Deutsch, P.T. Coleman et E.C. Marcus (Eds), The Handbook of Conflict Resolution: Theory and practice (p. 23-42). San Francisco : Jossey-Bass.

Fustier, P. (2012). Le lien d'accompagnement : un métissage entre échange par le don et échange contractualisé. Informations sociales, 1(169), 91-98. https://www.cairn.info/revueinformations-sociales-2012-1-page-91.htm

Hulin, A. (2010). Les pratiques de transmission du métier : de l'individu au collectif. Une application au compagnonnage. Thèse de doctorat en sciences de gestion, Université François-Rabelais, Tours.

Johnson, D.W. et Johnson, R.T. (1989). Cooperation and competition: Theory and research. Edina : Interaction.

Johnson, D.W. et Johnson, R.T. (2003). Social interdependence: The interrelationships among theory, research, and practice. The Center for Cooperative Learning, The University of Minnesota.

Larousse, Dictionnaire de français, repéré à http://www.larousse.fr/dictionnaires/francais/pairs Mauss, M. (1968). Essai sur le don. Sociologie et Anthropologie. Paris : Presses universitaires de France

Mazur, E. (1997). Peer Instruction : A User's Manual, Prentice Hall.

O’Donnell, A.M. et King, A. (1999). Cognitive Perspectives on Peer Learning. Mahwah : Lawrence Erlbaum Associates.

Paul, M. (2004). L'accompagnement : une posture professionnelle spécifique. Paris : L'Harmattan.

Paul, M. (2007). L'accompagnement ou la traversée des paradoxes. Dans J.P. Boutinet (dir.), Penser l'accompagnement des adultes - Ruptures, transitions, rebonds. (p. 251-274). Paris : PUF.

Thébault, J. (2013). La transmission professionnelle : processus d'élaboration d'interactions formatives en situation de travail. Une recherche auprès de personnels soignants dans un Centre Hospitalier Universitaire. Thèse de doctorat en Ergonomie, Creapt, CEE, CNAM Paris.

Thébault, J., Delgoulet, C., Fournier, P. S., Gaudart, C. et Jolivet, A. (2014). La transmission à l'épreuve des réalités du travail. Éducation permanente, 1(198), 85-99.

Wittorski, R. (1996), Évolution des compétences professionnelles des tuteurs par l'exercice du tutorat. Recherche et Formation, 22, 35-46. 


\section{Annexes \\ Annexe 1 : Questionnaire de satisfaction des étudiants-ingénieurs}

Total de 222 réponses de 2014 à 2016

1- Êtes-vous satisfait de la réactivité de votre coach?

2- Avez-vous eu besoin de relancer votre coach pour obtenir une réponse ?

3- Les conseils sur vos CV vous ont paru : pertinents, en phase avec vos attentes, ...

4- Combien d'échanges avez-vous réalisé ?

5- Pensez-vous avoir amélioré votre $\mathrm{CV}$ grâce à ce coaching ?

6- Qu'avez-vous appris en particulier?

7- Avez-vous bénéficié de conseils pour la journée des métiers ?

8- Avez-vous saisi cette opportunité pour demander des conseils sur la lettre de motivation?

9- Ce contact vous a-t-il permis de trouver un stage?

10- Avez-vous pensé à remercier votre coach de ses conseils ?

11-Quelles sont vos propositions d'améliorations ou attentes non contentées ?

\section{Résultats}

\section{Etes vous satisfait de la} réactivité de votre coach?

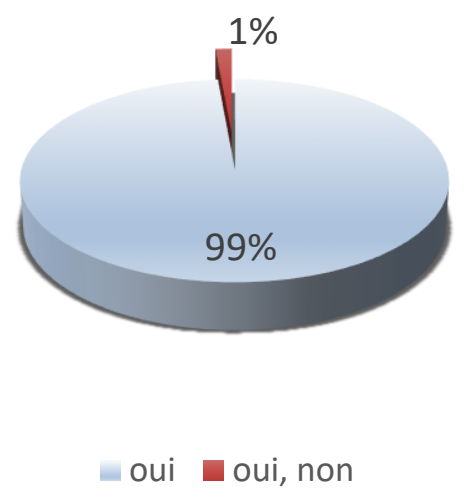

Avez -vous eu besoin de relancer votre coatch pour obtenir une réponse?

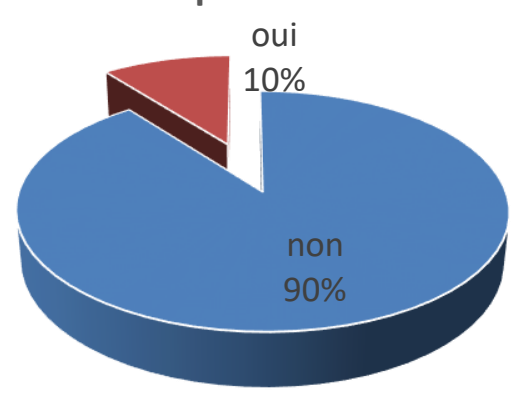

$$
\text { - non - oui }
$$


Pensez-vous avoir amélioré votre CV grâce à ce coaching ?

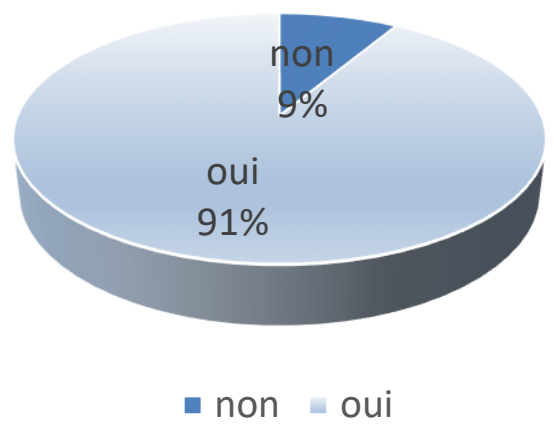

Avez-vous bénéficié de conseils pour la journée des métiers ?

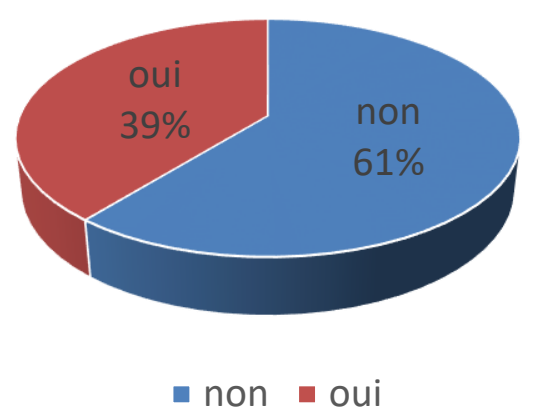

Avez-vous pensé à remercier votre coach de ses conseils?

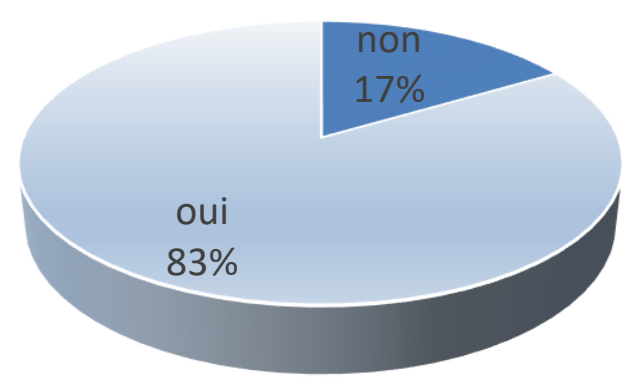

- non - oui
Avez-vous bénéficié de conseils pour la journée des métiers ?

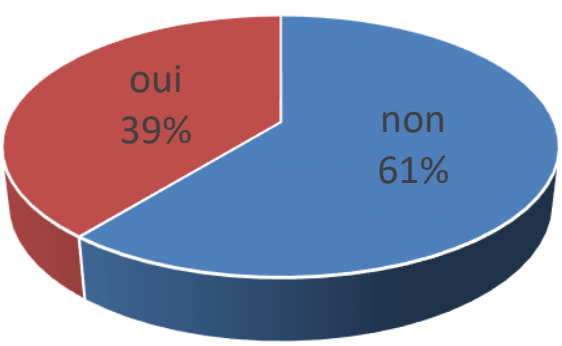

- non - oui

en phase avec vos attentes 


\title{
Qu'avez vous appris en particulier ?
}

\author{
synthétiser
}

Quelques points de mise en forme,... Pleins de détails, et des avis sur les questions..

Ordre des expériences pro mise en page

Mettre en avant mes "particularités" La mise en page

I'orientation du cv vers la qualité Informations importantes a mettre dans le... Écriture d'un CV plus pertinent et meilleur..

Comment ordonnancer les rubriques/ Attention à son image sur internet "Moderniser" mon CV

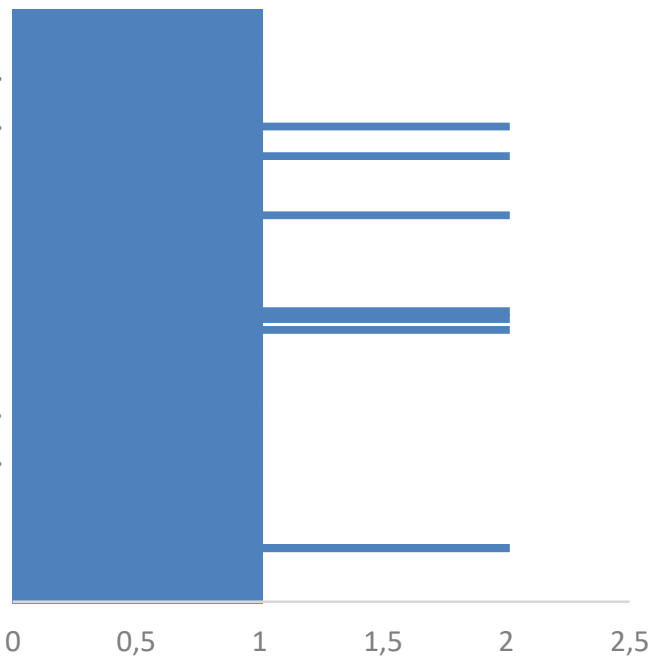


Annexe 2 : Retours sur le travail de Coach

\section{Bilan d'un étudiant de M2 RHOI 2015-2016}

Quels ont été les apports de cette expérience?

Cette expérience s'est révélée enrichissante pour ma part, j'y ai développé des compétences : transmission d'un savoir, gestion d'un groupe, acquisition d'un vocabulaire spécifique, capacité à être accessible, entendable de tous et pédagogue.

Transmettre son savoir demande de mobiliser ses connaissances, les organiser, les actualiser et les rendre entendables pour tous. J'ai adapté mon discours pour qu'il soit accessible au groupe et qu'il y ait une bonne compréhension de tous. Ce travail de coaching m'a semblé pertinent avec mon projet professionnel, car dans le futur, je serai amené à transmettre mes connaissances en m'assurant de la bonne compréhension de tous.

De plus, rencontrer les étudiants ingénieurs en Qualité et Sûreté de Fonctionnement m'a permis d'acquérir leurs vocabulaires spécifiques et une compréhension des compétences détenues par ces futurs ingénieurs.

Je souhaitais me rendre accessible pour l'autre, c'est pourquoi les étudiants pouvaient me contacter par mails pour toutes demandes d'informations, questionnements...

Enfin, j'ai nommé, avec bienveillance, ce qui dysfonctionnait ou ce qui n'était pas adapté dans leurs CV. Un positionnement parfois délicat à tenir mais nécessaire pour aider l'étudiant à réaliser un bon $\mathrm{CV}$.

\section{Quelles ont été les difficultés rencontrées ?}

Je me suis engagé avec ces étudiants dans le cadre du coaching et j'en ai mesuré la complexité. J'ai rencontré deux difficultés majeures :

- D'un point de vue technique, je réalise mon contrat de professionnalisation en HauteNormandie. J'habite dans un petit village sans connexion internet possible et le réseau téléphonique est variable, le contact avec les étudiants était par conséquent complexe.

- D'un point de vue méthodologique, j'ai eu peu d'échanges avec les étudiants. J'ai explicité précédemment mon choix concernant le fait de ne pas prendre le mail des étudiants. Néanmoins, lors de notre échange collectif, je leur ai fourni mon adresse mail afin que tous puissent bénéficier d'un accompagnement ciblé sur leurs besoins ( $\mathrm{CV}$ et divers). Ma démarche avait pour objet de les responsabiliser et de les laisser acteurs de leur projet. Je souhaitais leur laisser la possibilité de se saisir de l'aide que je pouvais leur apporter et non les contraindre.

\section{Quels ont été les éléments de satisfaction et d'insatisfaction?}

Ma principale satisfaction se situe au niveau du contact avec les étudiants. Lors du premier rendez-vous l'échange était constructif. Ils ont fait preuve d'une écoute attentive et ont pris beaucoup de notes. Lors de cette rencontre, ils ont posé de nombreuses questions et ont montré un véritable intérêt pour le sujet. De plus, les deux étudiants qui m'ont recontacté se sont avérés, une fois encore, à l'écoute des conseils et ils les ont appliqués. 
Mon insatisfaction se situe sur le faible nombre d'échanges avec les quatre autres étudiants. Je ne pense pas pour autant qu'il s'agit d'un manque d'implication, mais peut-être une difficulté d'organisation, un manque d'autonomie, un manque de contenance... Je pensais que leur laisser une certaine liberté susciterait chez eux une mobilisation plus forte qui selon moi s'avère être une première étape pour travailler en collaboration.

\section{Qu'est-ce que vous referiez de la même façon ou différemment ?}

Si cette expérience était à refaire, je modifierais ma conception du coaching. Je m'investirais différemment auprès d'eux: je n'attendrais pas que les étudiants viennent à moi, je les relancerais par mails, je donnerais des échéances... En effet, ma méthode de coaching n'a pas été productive sur l'ensemble des étudiants puisque seulement deux d'entre eux ont repris contact avec moi. C'est pourquoi, si j'avais l'occasion de recommencer, je fixerais des rencontres physiques ou virtuelles (skype) de manière à les motiver et à travailler plus efficacement.

\section{Quelles sont vos suggestions pour un travail plus satisfaisant ?}

Pour que le travail soit plus satisfaisant, je pense qu'il serait intéressant de prévoir d'autres rencontres officialisées sur les emplois du temps. Ces créneaux permettraient de faire le point régulièrement avec les étudiants. Effectivement, au vu du déroulement de l'expérience certains étudiants semblent avoir besoin d'un cadre contenant. Or, avoir des temps imposés, au même titre qu'un cours permet de penser en amont et en aval à la rencontre. Ces temps favoriseraient également la mise en place d'une dynamique de groupe. De plus, ces créneaux permettraient aux étudiants coachs, dans ma situation (contrat professionnalisation éloigné), de faciliter leurs échanges.

\section{Seriez-vous prêt à réitérer ce coaching (oui pourquoi, non pourquoi ?)}

Oui, avec plaisir! Même si je changerais probablement de méthodologie, ce coaching permet à chacun de grandir, d'apprendre des autres et de soi-même. En travaillant sur ce projet, j'ai renforcé mes compétences. Je pense que tout le monde est "gagnant» dans ce coaching, car cela permet un mixage intéressant entre deux formations qui ne sont, a priori, pas appelées à rentrer en contact. Ce type d'expérience est intéressant pour les masters professionnalisant comme le RHOI.

\section{Bilan d'une étudiante du M2 RHOI 2016-2017}

Tout d'abord, j'ai préparé cette séance de coaching de façon à apporter des conseils complémentaires à ceux qui ont été mis à la disposition des étudiants au préalable. Pour cela, j'ai préparé un PowerPoint reprenant les différents points du CV (identité, expériences, stages, formations compétences, loisirs).

De plus, j'ai pris le temps de faire des pauses entre chaque point pour répondre aux nombreuses questions et approfondir certains conseils. En fin de présentation, je me suis permis de faire un rappel sur l'importance des réseaux sociaux professionnels et des CV en ligne, qui peuvent être utilisés en complément du CV papier. Enfin, je les ai également sensibilisés sur l'impact, sur le recrutement que peuvent avoir les informations véhiculées via internet. Notamment en prenant à titre d'exemple des informations sur eux-mêmes que j'avais pu très facilement trouver. 
Suite à ma présentation, nous avons échangé par e-mail afin que je leur donne des conseils personnalisés sur chacun de leur CV. Pour ceux qui souhaitaient aller plus loin, j'ai également pu donner mon avis sur leur CV en ligne (Linkedin, Viadéo, Doyoubuzz).

Je pense que cette méthode de coaching a été plutôt efficace. Cela leur a permis d'améliorer leur CV en termes de fond, mais aussi de forme. De plus, ils ont pu comprendre la nécessité d'être présents sur les réseaux sociaux professionnels et de faire attention à la confidentialité des données personnelles qu'ils diffusent. Par ailleurs, je pense que j'aurais pu améliorer cette présentation en demandant leurs $\mathrm{CV}$ dès le début de la présentation pour avoir une approche plus personnalisée et pouvoir m'en servir comme support pour que mes propos aient peut-être encore plus d'impact.

Malgré cela, je pense que cette séance de coaching a été bénéfique autant aux étudiants qu'à moi-même. En effet, j'avais un peu d'appréhension avant d'effectuer cette présentation, dû au fait que je ne me sente pas forcément à l'aise à l'oral. Cependant, celle-ci s'est très vite dissipée lorsque j'ai commencé à répondre aux premières questions. Je me suis rendue compte que malgré mes doutes, je maîtrisais bien mon sujet grâce à mes différentes expériences dans le recrutement. Cela m'a permis par la suite d'avoir plus de confiance en moi, notamment dans l'exercice de mes missions actuelles en tant qu'assistante ressources humaines pour le Groupe SEB MOULINEX de Mayenne. En effet, je dois régulièrement faire des présentations et faire de l'événementiel.

De plus, échanger avec des étudiants de l'ISTIA était vraiment un plaisir. Ces derniers se sont montrés ouverts et chaleureux. J'ai ainsi pu découvrir des personnes faisant partie d'un univers professionnel différent du mien avec une culture pédagogique différente. Cela a été bénéfique pour mon expérience au sein du groupe SEB MOULINEX. En effet, nous avons beaucoup de personnes issues de formation ingénieur au sein du site et je suis amenée à rechercher régulièrement ce type de profil. J'ai ainsi pu en apprendre un peu plus sur les formations proposées dans cette filière et les compétences qui peuvent en découler.

Cependant, pour que ce coaching RH soit encore plus bénéfique aux étudiants, j'aurais préféré que la séance soit plus longue. Cela m'a obligé à priorisé en faisant une présentation plus générale et travailler les CV individuellement par e-mail.

Je pense que 2 heures auraient été préférables pour faire une partie de présentation et une seconde partie sur le travail des $\mathrm{CV}$ en commun. J'aurais ainsi pu proposer de commenter un par un les $\mathrm{CV}$ des étudiants pour que tout le monde puisse bénéficier des conseils. Je pense qu'avoir les personnes en face de soi pour les coacher est bien plus simple et cela a généralement plus d'impact. Pour ma part, je n'ai pas pu proposer de rendez-vous physiques à la suite de la présentation, car je ne suis pas du Maine-et-Loire. J'ai donc dû les coacher à distances par email, ce qui est plutôt impersonnel.

Pour autant, je serais ravie de réitérer l'expérience. Cela m'a beaucoup plu et j'ai eu le sentiment que cela avait vraiment eu un impact sur les étudiants. J'ai beaucoup aimé échanger avec eux et pouvoir leur donner les conseils et astuces que je connaissais du fait de mon expérience. Je suis consciente que même un recruteur expérimenté peut évincer un bon candidat pour la simple raison que son $\mathrm{CV}$ n'est pas bien fait. Il est très important de bien travailler son $\mathrm{CV}$ pour que chaque expérience 


\section{Annexe 3 : Récapitulatif du travail réalisé par les coachs M2 avec les}

étudiants-ingénieurs

\begin{tabular}{|c|c|c|c|c|c|c|}
\hline $\begin{array}{c}\text { Nom et } \\
\text { Prénom de } \\
\text { l'étudiant } \\
\text { d'EI4 } \\
\end{array}$ & Étudiant 1 & Étudiant 2 & Étudiant 3 & Étudiant 4 & Étudiant 5 & Étudiant 6 \\
\hline Spécialité & Innovation & Innovation & $\begin{array}{l}\text { Automatique et } \\
\text { Génie Info }\end{array}$ & $\begin{array}{l}\text { Automatique et } \\
\text { Génie Info }\end{array}$ & $\begin{array}{l}\text { Qualité et Sûreté } \\
\text { de } \\
\text { Fonctionnement }\end{array}$ & $\begin{array}{c}\text { Automatique et } \\
\text { Génie } \\
\text { Informatique }\end{array}$ \\
\hline $\begin{array}{c}\begin{array}{c}\text { Nombre } \\
\text { total } \\
\text { d'échanges }\end{array} \\
\end{array}$ & 4 & 3 & 3 & 4 & 4 & 1 \\
\hline $\begin{array}{c}\text { Prise de } \\
\text { contact avec } \\
\text { vous } \\
\text { satisfaisante } \\
?\end{array}$ & & & Oui & our tous & & \\
\hline $\begin{array}{c}\text { Conseils } \\
\text { principaux } \\
\text { donnés à } \\
\text { l'étudiant }^{5}\end{array}$ & $\begin{array}{l}\text { 1) Mettre le } \\
\text { CV en format } \\
\text { PDF } \\
\text { 2) Faire des } \\
\text { liens cliquables } \\
\text { du CV vers ses } \\
\text { CV en ligne } \\
\text { 3) Faire } \\
\text { ressortir le titre } \\
\text { du CV } \\
\text { 4) Mettre ses } \\
\text { coordonnées } \\
\text { sur le côté } \\
\text { 5) Identifier } \\
\text { des titres de } \\
\text { postes pour ses } \\
\text { stages } \\
\text { 6) Regrouper } \\
\text { par catégories } \\
\text { les } \\
\text { compétences } \\
\text { 7) Valoriser } \\
\text { exp.étudiantes }\end{array}$ & $\begin{array}{l}\text { 1) Mettre le } \\
\text { CV en format } \\
\text { PDF } \\
\text { 2) Changer son } \\
\text { adresse mail } \\
\text { qui est celle de } \\
\text { l'ISTIA } \\
\text { 3) Préciser son } \\
\text { option dans sa } \\
\text { formation } \\
\text { 4) Conseils de } \\
\text { mise en forme } \\
\text { du CV (tirets, } \\
\text { espace, } \\
\text { retraits...) } \\
\text { 5) Créer une } \\
\text { adresse mail } \\
\text { plus } \\
\text { professionnelle }\end{array}$ & $\begin{array}{l}\text { 1) Mettre ses } \\
\text { coordonnées sur } \\
\text { le côté et } \\
\text { uniquement le } \\
\text { titre du CV au } \\
\text { milieu } \\
\text { 2) Changer } \\
\text { « Technicien } \\
\text { supérieur » en } \\
\text { « Technicien } \\
\text { BE » }\end{array}$ & $\begin{array}{c}\text { 1) Mettre } \\
\text { « expériences } \\
\text { professionnelles » } \\
\text { plutôt que } \\
\text { « expériences } \\
\text { personnelles » } \\
\text { 2) Attribuer des } \\
\text { niveaux types B1, } \\
\text { B2 pour les } \\
\text { langues et non } \\
\text { plus « scolaire », } \\
\text { " intermédiaire ». } \\
\text { 3) Valoriser ses } \\
\text { projets extra- } \\
\text { scolaires } \\
\text { 4) Mettre plus en } \\
\text { avant le titre du } \\
\text { CV plutôt que } \\
\text { "stage de } 3 \\
\text { mois » }\end{array}$ & $\begin{array}{l}\text { 1) Ne pas mettre } \\
\text { " Etudiant» } \\
\text { dans le titre mais } \\
\text { plutôt identifier } \\
\text { un titre de poste } \\
\text { 2) Repréciser sa } \\
\text { formation à } \\
\text { Madagascar } \\
\text { 3) Être plus } \\
\text { précis dans les } \\
\text { dates de ses } \\
\text { stages } \\
\text { 4) Retirer les } \\
\text { ". »à la fin de } \\
\text { chaque phrase }\end{array}$ & $\begin{array}{l}\text { Je n'ai plus son } \\
\text { premier CV donc } \\
\text { je ne peux pas me } \\
\text { prononcer }\end{array}$ \\
\hline $\begin{array}{c}\text { Le CV a-t-il } \\
\text { évolué suite } \\
\text { à vos } \\
\text { conseils }\end{array}$ & \multicolumn{5}{|c|}{ Oui, tout à fait } & Aucun retour \\
\hline
\end{tabular}

\footnotetext{
${ }^{4}$ Échanges $=$ nombre de mails reçus

${ }^{5}$ Conseils donnés suite aux CV retravaillés car les premiers CV ne sont plus en ma possession
} 


\begin{tabular}{|c|c|c|c|c|c|c|}
\hline $\begin{array}{c}\text { Quels } \\
\text { conseils } \\
\text { avez-vous } \\
\text { prodigués } \\
\text { au-delà du } \\
\text { CV? }\end{array}$ & \multicolumn{6}{|c|}{$\begin{array}{l}\text { 1) Utiliser le carré des compétences pour les aider à mieux se connaître et à construire leurs projets professionnels } \\
\text { (carré que j'ai proposé à la première réunion) car c'est ce qui leur permettra de se différencier durant les entretiens } \\
\text { de recrutement } \\
\text { 2) Pour construire son projet professionnel, ne pas hésiter à aller regarder les fiches métiers existantes sur internet } \\
\text { 3) Intégrer la notion de « compétences » dans leurs CV et lettres de motivation : que savez-vous ? que savez-vous } \\
\text { faire? quel degré de maîtrise pour chaque compétence? } \\
\text { 4) Pour ceux qui le souhaitent, ne pas hésiter à utiliser et exploiter les réseaux professionnels tels que Linkedin } \\
\text { et/ou Viadeo pour être plus visible (e-recrutement) } \\
\text { 4) Pour Léo } \rightarrow \text { correction de sa lettre de motivation pour un stage }\end{array}$} \\
\hline $\begin{array}{l}\text { Défauts } \\
\text { récurrents } \\
\text { repérés }\end{array}$ & $X$ & $X$ & $X$ & $\begin{array}{c}\text { Il insistait pour } \\
\text { mettre } \\
\text { « expériences } \\
\text { personnelles » } \\
\text { dans son CV } \\
\end{array}$ & $X$ & $X$ \\
\hline $\begin{array}{c}\text { Votre niveau } \\
\text { de } \\
\text { satisfaction } \\
\text { sur vos } \\
\text { échanges : } \\
\end{array}$ & \multicolumn{5}{|c|}{$\begin{array}{l}\text { Très satisfaite, échanges constructifs, étudiants impliqués et à l'écoute de mes conseils. Ils } \\
\text { ont très bien travaillé. }\end{array}$} & $\begin{array}{c}\text { Satisfaite dans les } \\
\text { premiers } \\
\text { échanges mais } \\
\text { plus de nouvelles } \\
\text { ensuite, } \\
\text { dommage... }\end{array}$ \\
\hline $\begin{array}{c}\text { Autres } \\
\text { remarques }\end{array}$ & \multicolumn{6}{|c|}{$X$} \\
\hline
\end{tabular}

